\title{
Colon Stricture After Ischemia Following a Robot-Assisted Ultra-Low Anterior Resection With Coloanal Anastomosis
}

\author{
Dae Ro Lim, Hyuk Hur, Byung Soh Min, Seung Hyuk Baik, Nam Kyu Kim \\ Section of Colon and Rectal Surgery, Department of Surgery, Yonsei University College of Medicine, Seoul, Korea
}

Four consecutive cases of a colonic stricture following a da Vinci robot-assisted ultra-low anterior resection (LAR) with coloanal anastomosis and diverting ileostomy for the treatment of rectal cancer are reported. The colonic strictures developed after early proximal colonic ischemia without anastomotic site leakage or disruption. All patients were treated with preoperative chemoradiation therapy. During the postoperative recovery period, patients developed colonic ischemia, presenting with a high, spiking fever, but without any symptoms of peritonitis. Patients were treated with conservative management (antibiotic therapy) and discharged after two weeks when in good condition. Several months after discharge, all four patients developed a long-segment colonic stricture from the anastomosis site to the distal colon. Management of the colon strictures, including the anastomotic site, involved colonic dilation with a Hegar dilator in an outpatient clinic for several months. The ileostomies in three patients could not be closed.

Keywords: Colonic stricture; Colonic ischemia; Coloanal anastomosis surgery

\section{INTRODUCTION}

Colonic stricture following major vascular surgery (e.g., surgery for an aortic aneurism) is common, and many cases have been reported. However, colonic stricture following colorectal surgery is rare and may be attributed to a number of factors, including suturing techniques, leakage, colonic ischemia, and radiotherapy [1, 2]. Edema from colonic ischemia and fibrosis is considered the main mechanism leading to colonic stricture [3]. Few studies have addressed colonic ischemia following colorectal surgery, and colonic stricture after colonic ischemia is rarely reported [4]. The present study describes four cases of colonic stricture that occurred after early colonic ischemia following a robot-assisted ultra-low anterior resection (LAR) and coloanal anastomosis for the treatment of low rectal cancer.

Received: January 2, 2013 Accepted: June 22, 2015

Correspondence to: Nam Kyu Kim, M.D.

Section of Colon and Rectal Surgery, Department of Surgery, Yonsei University College of Medicine, 50 Yonsei-ro, Seodaemun-gu, Seoul 03722, Korea

Tel: +82-2-2228-2105, Fax: +82-2-313-8289

E-mail:namkyuk@yuhs.ac

(C) 2015 The Korean Society of Coloproctology

This is an open-access article distributed under the terms of the Creative Commons Attribution NonCommercial License (http://creativecommons.org/licenses/by-nc/3.0) which permits unrestricted noncommercial use, distribution, and reproduction in any medium, provided the original work is properly cited.

\section{CASE REPORTS}

All patients underwent a robot-assisted ultra LAR with coloanal anastomosis for the treatment of rectal cancer and a diverting loop ileostomy. They were also treated with preoperative chemoradiation therapy (total of 50.4 Gy, 5-fluorouracil-based chemotherapy). The pathologic results of rectal tumors after biopsy using colonoscopy were consistent with an adenocarcinoma in all cases.

\section{Case 1}

The patient was a 57 -year-old man with a tumor located $3 \mathrm{~cm}$ from the anal verge. The sigmoidal artery was ligated and the left colic artery was preserved after a frozen biopsy of an inferior mesenteric artery node (low ligation). Pathology findings revealed a Mandard grade-III ypT2N0M0 lesion. On postoperative day (POD) 4 , the patient developed a high, spiking fever $\left(39.2^{\circ} \mathrm{C}\right)$, but no other signs of anastomosis site leakage or peritonitis were found. On POD 7, the high, spiking fever remained, so an abdominal pelvic computed tomography (CT) was subsequently performed. The CT scan showed ischemia of the distal colon from the coloanal anastomosis site to the proximal colon, about $15 \mathrm{~cm}$ (Fig. 1). On sigmoidoscopy, the mucosa color was intact, and no other specific findings were noted. Antibiotic therapy continued until POD 18. The patient was discharged with good condition on POD 21. Approximately seven months after his dis- 


\section{Annals of Colon Stricture After Ischemia Following a Robot-Assisted Ultra-Low Anterior Resection With Coloanal}

Coloproctology Dae Ro Lim, et al.
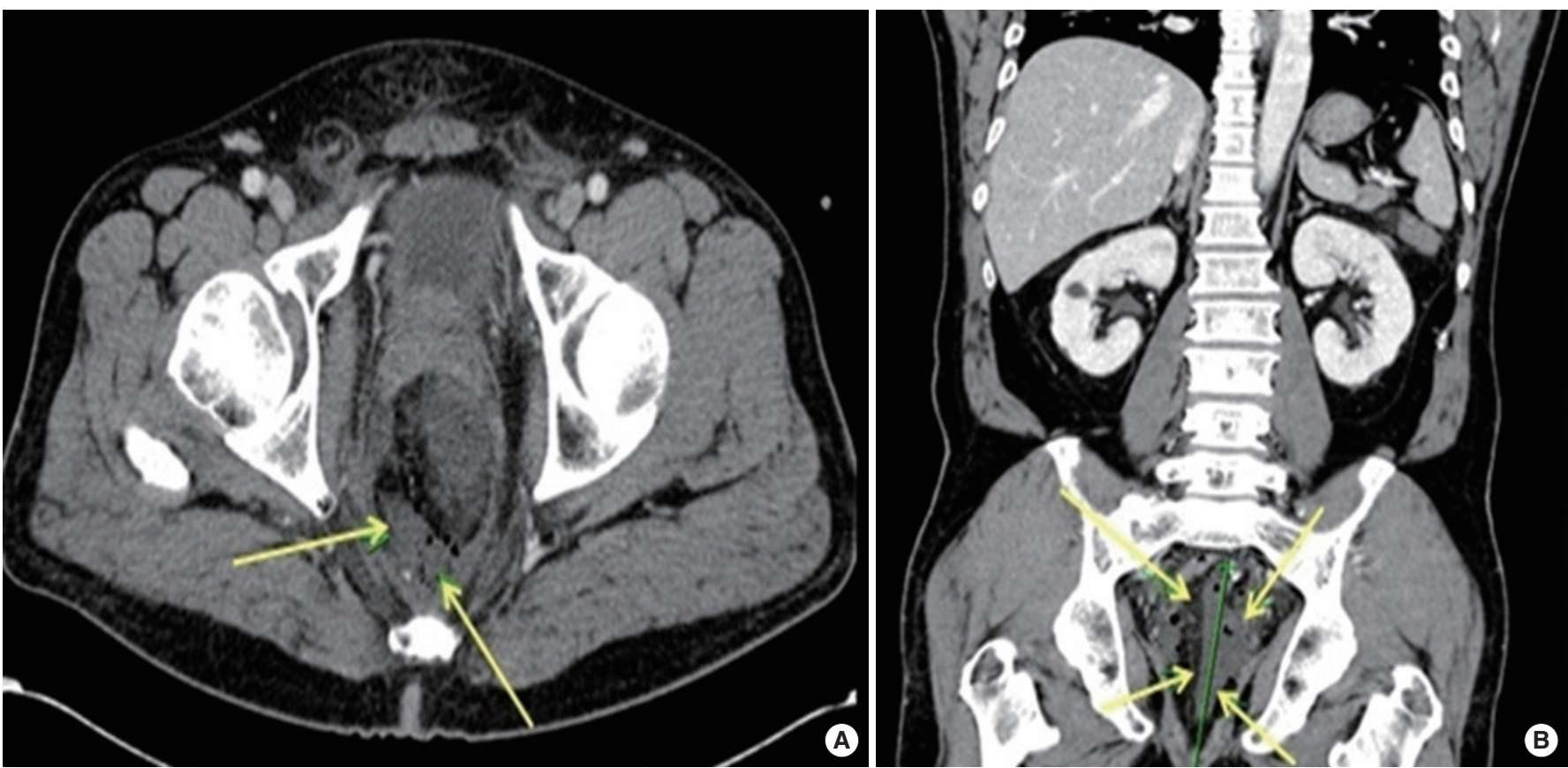

Fig. 1. Case 1: (A) a computed tomography scan featuring a small amount of fluid with air bubbles in the presacral area (arrows), (B) decreased perfusion of the distal colon (anal verge $15 \mathrm{~cm}$ from the coloanal anastomosis site), and ischemia of infarction (arrows).
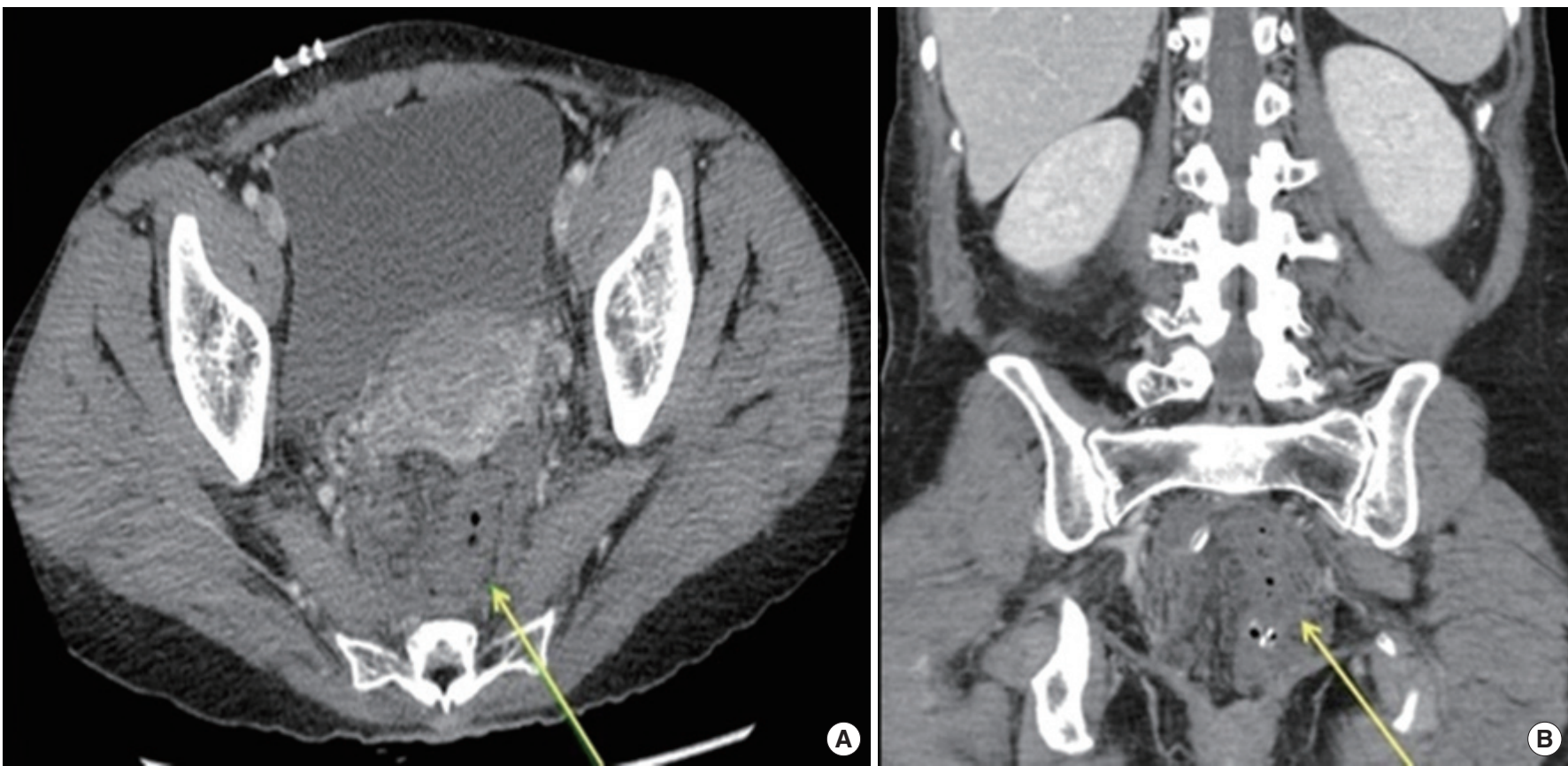

Fig. 2. Case 2: (A) a markedly decreased perfusion and edematous bowel wall thickening from the midsigmoid colon to the anus with fatty stranding (arrow) (transverse plan view), (B) suggesting a necrotic change (arrow) (coronal view).

charge, the patient developed an anastomotic stricture. The stricture was subsequently managed at an outpatient clinic by dilation with a Hegar dilator.
Case 2

The patient was a 54-year-old woman with a tumor located $3 \mathrm{~cm}$ from the anal verge. The patient underwent low ligation. Pathol- 

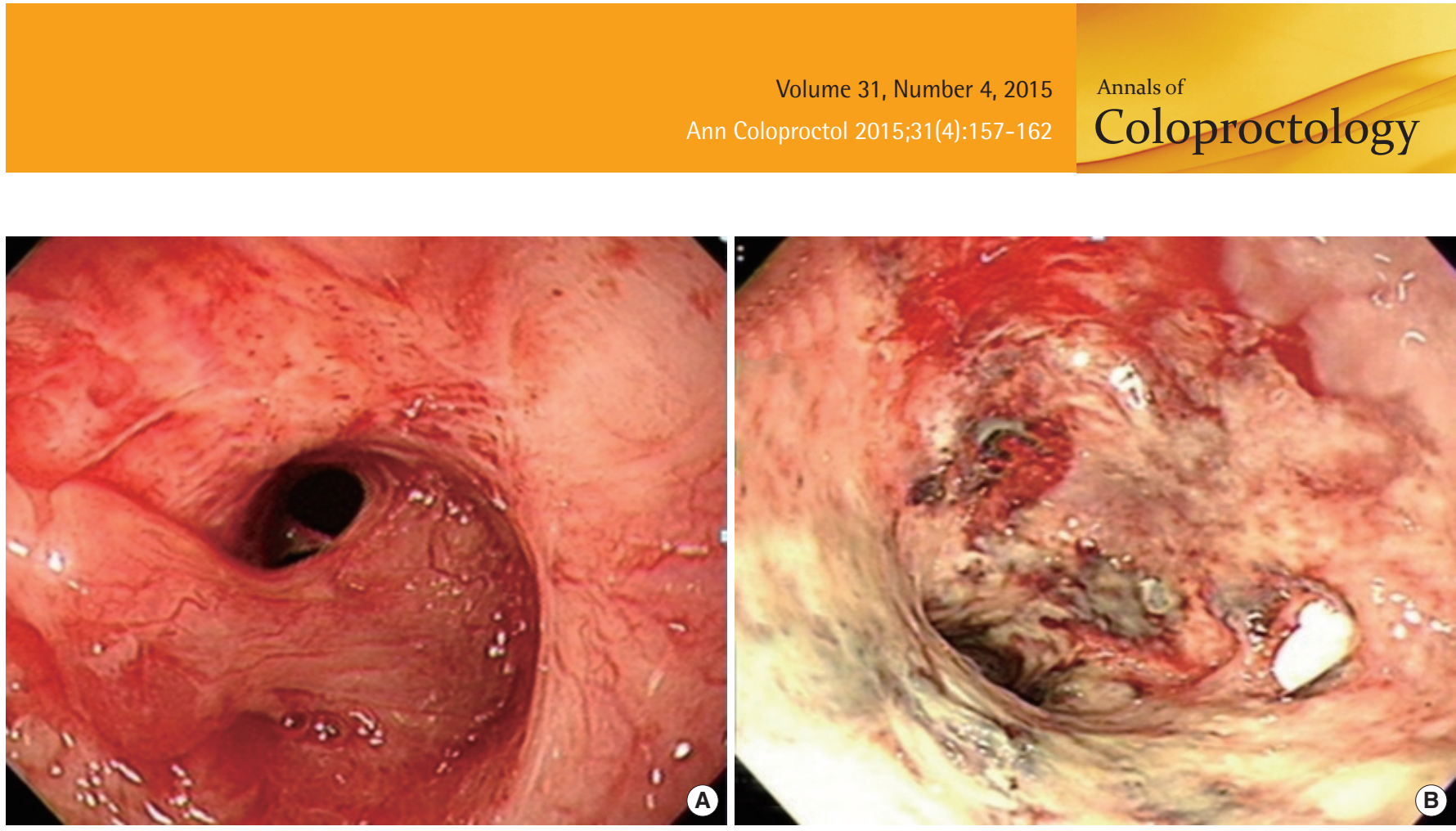

Fig. 3. Case 2: (A) colonoscopy showing a colonic stricture (anal verge $5 \mathrm{~cm}$ ), (B) bleeding upon slight touching and inflammatory changes.
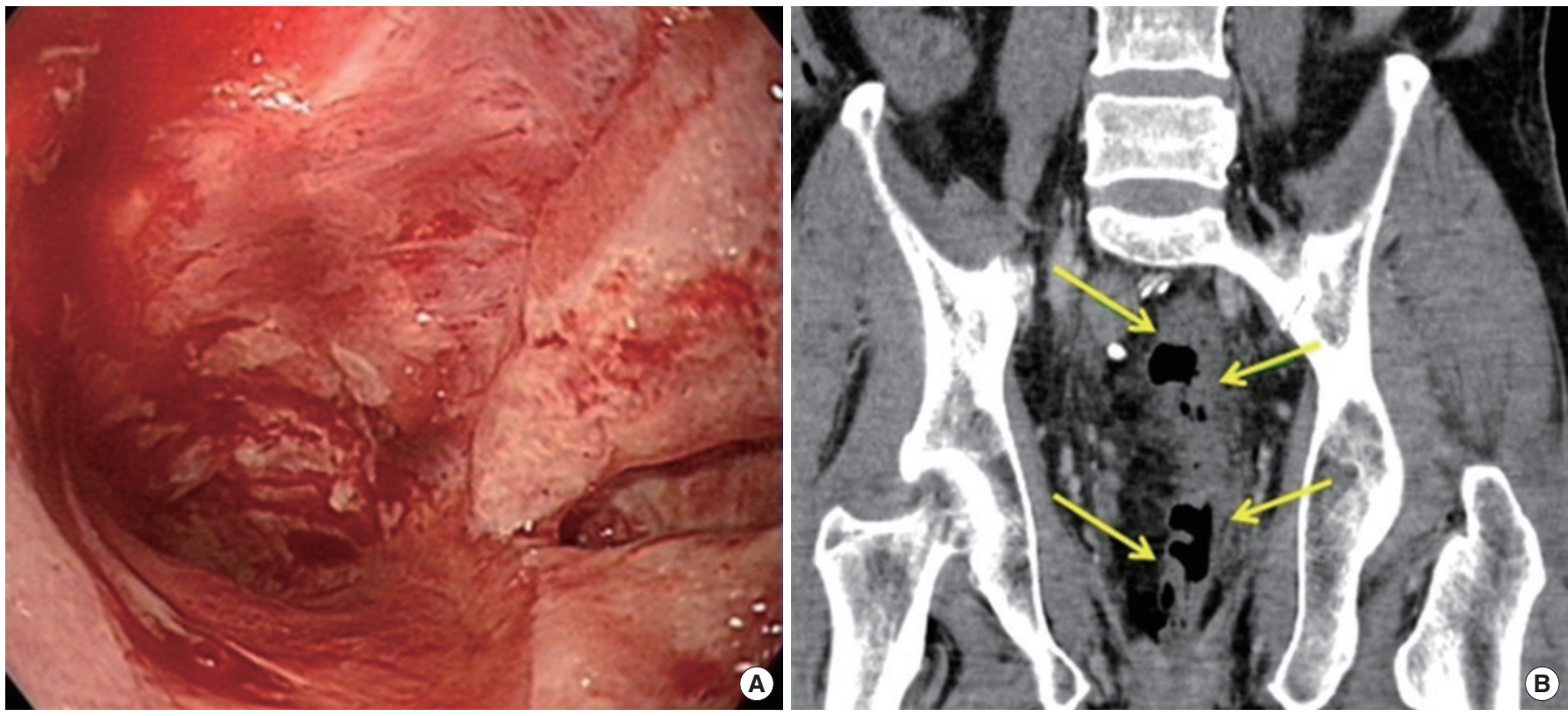

Fig. 4. Case 3: colonoscopy featuring colonic ischemia (A) and computed tomography (B) demonstrating ischemic changes of the distal colonanus (arrows) (about $15 \mathrm{~cm}$ ).

ogy findings revealed a Mandard grade-I ypTxN0M0 lesion. On POD 5, the patient developed a high, spiking fever $\left(38.2^{\circ} \mathrm{C}\right)$, but no signs of leakage were noted. A CT scan featured markedly decreased perfusion and edematous bowel wall thickening from the mid sigmoid colon to the anus, suggesting ischemic change (Fig. 2). However, on sigmoidoscopy, the mucosa color was intact, and no other specific findings were noted. Therefore, conservative treatment was undertaken, and antibiotic therapy continued until POD 13. The patient was discharged with good condition on POD
15. About six months after discharge, she developed a colonic stricture. Colonoscopy revealed a stricture $5 \mathrm{~cm}$ from the anal verge, and the scope could not pass through (Fig. 3). The stricture was subsequently managed at an outpatient clinic by dilation with a Hegar dilator. Approximately six months after discharge, the patient underwent ileostomy closure and colonic dilation with a Hegar dilator in the operation room. She was discharged after being put on a tolerable diet and had passed a small-caliber stool with frequent bowel movements. 

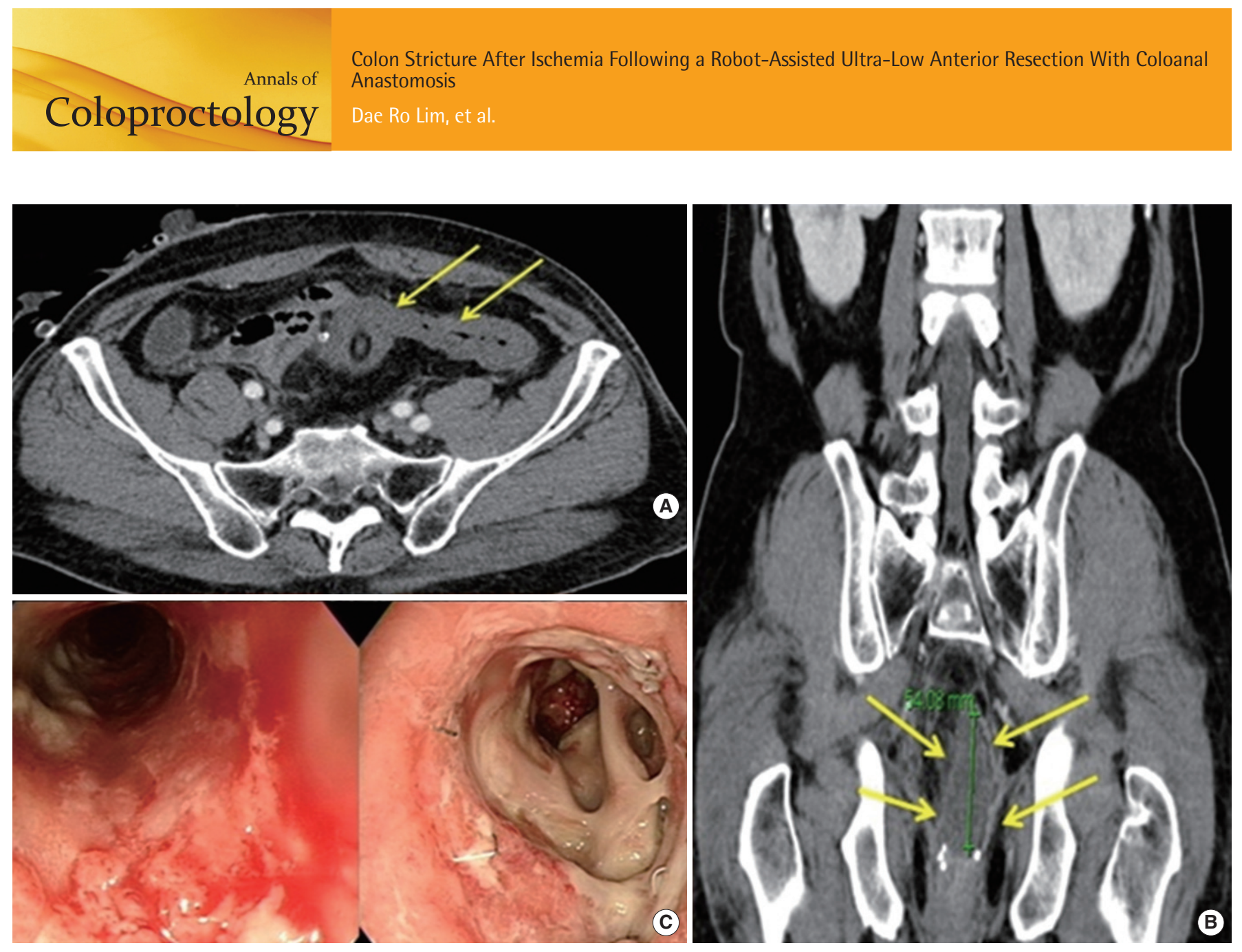

Fig. 5. Case 4: (A) postoperative day 1, computed tomography (CT) findings of ischemic colitis (arrows), (B, C) postoperative 3 weeks, CT findings demon (arrows) and colonoscopic findings demonstrating colonic ischemia and infarction.

\section{Case 3}

The patient was a 63-year-old man with a tumor located $5 \mathrm{~cm}$ from the anal verge. He underwent low ligation. Pathology findings revealed a Mandard grade-III ypT3N1bM0 lesion. On POD 2, the patient developed a high, spiking fever $\left(38.6^{\circ} \mathrm{C}\right)$, but had no signs of leakage or peritonitis. A CT scan featured slightly decreased perfusion and edematous bowel wall thickening from the distal colon to the anus, suggesting ischemic change of a $15-\mathrm{cm}$ long portion of the distal colon-anus (Fig. 4). On sigmoidoscopy, the mucosa color was intact, and no other specific findings were noted. Therefore, conservative treatment was undertaken, and antibiotic therapy continued until POD 11. The patient was discharged to his home in a satisfactory condition on POD 11. Approximately six months after discharge, colonoscopy revealed ischemic colitis $15 \mathrm{~cm}$ in length from the anastomosis site to the distal colon (Fig. 4). Erythema, hyperemia, exudates, petechiae, and mucosal friability of the colon lumen were present. Approximately 13 months after discharge, he developed a colonic stricture.

\section{Case 4}

The patient was a 60 -year-old man with a tumor located $5 \mathrm{~cm}$ from the anal verge. The patient underwent high ligation of the inferior mesenteric artery. Pathology findings revealed a Mandard grade-III, ypT3N2M0 lesion. On POD 1, the patient developed a high, spiking fever $\left(38.7^{\circ} \mathrm{C}\right)$, but had no signs of leakage or peritonitis. A CT scan featured slightly decreased perfusion and edematous bowel wall thickening from the proximal colon (Fig. 5). The patient was treated with antibiotic therapy and discharged with good condition on POD 10. Approximately two weeks after discharge, he was readmitted due to a high, spiking fever and abdominal pain. A CT finding revealed ischemic colitis and near infarction from the anastomosis site to the distal colon, a length of more than $6 \mathrm{~cm}$ (Fig. 5). Colonoscopy revealed erythema, hyperemia, exudates, petechiae, healing ulceration, and mucosal friability of the colon lumen. After antibiotic therapy for two weeks, the patient was discharged. Approximately three months later, he developed a colonic stricture.

\section{DISCUSSION}

Colonic stricture after a LAR for treating patients with rectal cancer has been reported in up to six percent of patients [4], and a six percent incidence of large bowel strictures after preoperative radiotherapy has also been reported [5]. In the present cases, the 
four patients developed colonic ischemia during the postoperative recovery after a robot-assisted ultra-LAR and coloanal anastomosis. A characteristic of this colonic ischemia was that it occurred over a long segment of tissue, about 6-15 cm from the site of anastomosis. Following treatment of this colonic ischemia, the patients developed a colonic stricture. Colonic ischemia following colorectal surgery is a rare, but serious, complication. It is usually the result of a sudden and temporary reduction in blood flow that is insufficient to meet the metabolic demands of discrete regions of the colon. From a review of the literature, the risk factors associated colonic ischemia following colorectal surgery are radiation therapy [4], high ligation of the inferior mesenteric artery [6], old age, and cardiovascular disease [7]. In the present cases, all patients had undergone preoperative chemoradiation therapy. Three patients underwent low ligation of the inferior mesenteric artery. Additionally, the ages of all the patients fell between the fifth and the early sixth decades, and none had cardiovascular disease. We, therefore, posit that preoperative radiation therapy may play a large role in the development of colonic ischemia. Preoperative chemoradiation therapy may sometimes cause bowel damage and enteritis. A redundant sigmoid colon inside the pelvic cavity may be damaged by radiation during preoperative therapy. Therefore, one possible way of preventing colonic ischemia is to ensure resection of a sufficient portion of the sigmoid colon that had received chemoradiation. To do this, adequate splenic flexure mobilization is necessary. However, differentiating between the normal bowel and radiation-damaged bowel by gross inspection at the time of surgery is very difficult. Thus, the status of the bowels must be checked carefully in preoperative imaging studies following chemoradiation therapy.

Another factor contributing to colonic ischemia is insufficient blood supply. After coloanal anastomosis, colon tension can give rise to insufficient blood supply to the colon, and colonic ischemia can develop. Therefore, when the specimen is retrieved through the anus, the tension of the remaining distal bowel must be monitored through the intra-abdomen by using laparoscopy. If there is distal colon tension after the specimen is pulled out, the distal colon must be lengthened or mobilized adequately. Another reason for insufficient blood supply occurs at the time of the ligation of the inferior mesenteric vein. A collateral artery sometimes runs with the inferior mesenteric vein, and if the collateral artery is ligated together when the inferior mesenteric vein is ligated, this can result in insufficient blood supply to the distal colon. Therefore, the inferior mesenteric vein should be carefully skeletonized during ligation. The limited collateralization of vasculature that occurs at the rectosigmoid junction may be an additional contributing factor. Nonocclusive colonic ischemia most commonly affects the watershed areas of the colon that have limited collateralization, such as the splenic flexure and the rectosigmoid junction $[8,9]$.

Management of colonic ischemia, which presented in all the patients in the current study, was conservative (i.e., antibiotic therapy), and management of the anastomotic stricture was by transanal dilations. Generally, management of an anastomotic stricture involves Hegar dilators, stricture scopes [10] and Foley catheter balloons [11], though endoscopic balloon dilation has been used [12]. For the patients in the present study, we used a Hegar dilator to perform colonic dilation at an outpatient clinic for several weeks to months. However, with this method, ileostomy closure was possible for just one of the four patients. The basic management of the colonic ischemia is to remove the ischemic colon and recoloanal anastomosis by using the remaining descending colon again.

In summary, we found four cases of colonic strictures, which were preceded by early colonic ischemia and occurred following a robot-assisted ultra-LAR with coloanal anastomosis and diverting ileostomy for the treatment of patients with rectal cancer. Management for these cases involved the use of a Hagar dilator at an

Table 1. Summary of patients

\begin{tabular}{|c|c|c|c|c|c|c|c|}
\hline Age $(y r) / s e x$ & $\begin{array}{c}\text { Rectal cancer } \\
\text { distance from AV }\end{array}$ & Preoperative CCRTx & Operation name & IMA ligation & Interval event & Symptom & CT finding \\
\hline $57 / M$ & $3 \mathrm{~cm}$ & $\mathrm{FL}^{*}+50.4 \mathrm{~Gy}$ & R-uLAR CAA & Low & POD 4 & Fever $\left(39.2^{\circ} \mathrm{C}\right)$ & Colonic ischemia \\
\hline $54 / F$ & $3 \mathrm{~cm}$ & $\mathrm{FL}+50.4 \mathrm{~Gy}$ & R-uLAR CAA & Low & POD 5 & Fever $\left(38.2^{\circ} \mathrm{C}\right)$ & Colonic ischemia \\
\hline $63 / \mathrm{M}$ & $5 \mathrm{~cm}$ & TS1 + irrinotecan + 50.4 Gy & R-uLAR CAA & Low & POD 2 & Fever $\left(38.6^{\circ} \mathrm{C}\right)$ & Colonic ischemia \\
\hline $60 / M$ & $5 \mathrm{~cm}$ & $\mathrm{FL}+48.6 \mathrm{~Gy}$ & R-uLAR CAA & High & POD 1 & Fever $\left(38.7^{\circ} \mathrm{C}\right)$ & Colonic ischemia \\
\hline
\end{tabular}

AV, anal verge; CCRTx, concurrent chemoradiation therapy; IMA, inferior mesenteric artery; CT, computed tomography; FL, fluorouracil-leucovorin; R-uLAR CAA, robot-assisted ultra-low anterior resection with coloanal anastomosis; POD, postoperative day.

Table 2. Treatment of patients

\begin{tabular}{lccccc}
\hline Case & Treatment of colonic ischemia & Discharge day & Time of colonic stricture after discharge & Treatment of colonic stricture & Closure of ileostomy \\
\hline 1 & Conservative management (antibiotics) & POD 21 & 7 Months & Hegar dilator & No \\
2 & Conservative management (antibiotics) & POD 15 & 6 Months & Hegar dilator & Hegar dilator \\
3 & Conservative management (antibiotics) & POD 11 & 6 Months & Hegar dilator & No \\
4 & Conservative management (antibiotics) & POD 10 & 3 Months \\
\hline
\end{tabular}

POD, postoperative day. 
outpatient clinic, but ileostomy closure could not be performed (Tables 1, 2). In conclusion, for patients undergoing a robot-assisted ultra-LAR for the treatment of distal rectal cancer after chemoradiation therapy, colonic ischemia is a serious and real possibility. Although the condition of the bowel may seem normal during surgery, the possibility of delayed colonic ischemia always exists. If colonic ischemia is to be avoided, the issues we addressed before should be carefully considered during surgery.

\section{CONFLICT OF INTEREST}

No potential conflict of interest relevant to this article was reported.

\section{REFERENCES}

1. Senagore A, Milsom JW, Walshaw RK, Dunstan R, Mazier WP, Chaudry IH. Intramural $\mathrm{pH}$ : a quantitative measurement for predicting colorectal anastomotic healing. Dis Colon Rectum 1990; 33:175-9.

2. Orsay CP, Bass EM, Firfer B, Ramakrishnan V, Abcarian H. Blood flow in colon anastomotic stricture formation. Dis Colon Rectum 1995;38:202-6.

3. Brandt LJ, Boley SJ. Colonic ischemia. Surg Clin North Am 1992;
72:203-29.

4. Pahlman L, Glimelius B, Frykholm G. Ischaemic strictures in patients treated with a low anterior resection and perioperative radiotherapy for rectal carcinoma. Br J Surg 1989;76:605-6.

5. Law WL, Choi HK, Chu KW, Tung HM. Radiation stricture of rectosigmoid treated with self-expanding metallic stent. Surg Endosc 2002;16:1106-7.

6. Bernstein WC, Bernstein EF. Ischemic ulcerative colitis following inferior mesenteric arterial ligation. Dis Colon Rectum 1963;6:5461.

7. Sreenarasimhaiah J. Diagnosis and management of ischemic colitis. Curr Gastroenterol Rep 2005;7:421-6.

8. Gandhi SK, Hanson MM, Vernava AM, Kaminski DL, Longo WE. Ischemic colitis. Dis Colon Rectum 1996;39:88-100.

9. Greenwald DA, Brandt LJ, Reinus JF. Ischemic bowel disease in the elderly. Gastroenterol Clin North Am 2001;30:445-73.

10. Dencker H, Johansson JI, Norryd C, Tranberg KG. Dilator for treatment of strictures in the upper part of the rectum and the sigmoid. Dis Colon Rectum 1973;16:550-2.

11. Mazier WP. A technic for the management of low colonic anastomotic stricture. Dis Colon Rectum 1973;16:113-6.

12. Kan H, Furukawa K, Suzuki H, Tajiri T. Endoscopic balloon dilation therapy for postoperative anastomotic stenosis after colorectal resection. J Colon Exam 2006;23:21-3. 